\title{
NIVEL DE RENDIMIENTO ACADÉMICO, DESERCIÓN ESTUDIANTIL E INDICE DE MATRÍCULA EN LA FACULTAD DE OBSTETRICIA - UNJBG 2004
}

\author{
Responsable : Mgr. Rina Alvarez Becerra \\ Miembro : Lic. Educ. Gladys Benitez Palacios
}

\begin{abstract}
RESUMEN
El presente trabajo tuvo como objetivo determinar el nivel de rendimiento académico, el indice de matrícula y el indice de deserción de los estudiantes de la Facultad de Obstetricia durante el año académico 2004 en la Universidad Nacional Jorge Basadre Grohmann de Tacna. El diseño de investigación es no experimental, transversal descriptivo. Se ha utilizado como instrumento de recolección de datos un cuestionario ad hoc.

En conclusión el rendimiento académico en el año 2004 . 2005 fue de 12,53, valor que se ubica en la categoria de regular, al igual que la mayoria de los promedios obtenidos por año de estudios. El indice de deserción fue de 6 y las materias más reprobadas corresponden a cursos de formación general que se ubican en el primer año de estudios.
\end{abstract}

\begin{abstract}
The present research had the objective to determinate the academic performance level, the registration index and the desertion index of the Obstectric Faculty students at the Nacional University of Tacna.The design of the investigation is no experimental, descriptive and transversal. The instrument to collect data was an questionnaire ad hoc.

As a conclusion, the academic performance was $12,52 \%$ in 2004 . This value was place in the regular category. Also the majority of the averages obtained per year, were in the same category. The desertion index was six, and the subject that students frequently failed was General Studies during the First Grade.
\end{abstract}

\section{INTRODUCCIÓN}

Respecto al concepto de rendimiento académico, no existe un consenso, presentándose más bien como un concepto multidimensional, relativo y contextual. Lo habitual es analizar el rendimiento académico en términos de resultado, distinguiendo dos categorías: resultados inmediatos (calificaciones obtenidas durante el proceso formativo para el logro de su titulación) o rendimiento interno y resultados diferidos o rendimiento externo (impacto que la formación tiene sobre la vida social o sobre su incorporación a la vida laboral).

Por otro lado, el rendimiento académico tiene niveles. Los estudiantes normales, que son agentes principales de su educación, pueden alcanzar por sí mismos un rendimiento alto, medio o bajo. Hay alumnos que cuando presentan bajo rendimiento desaprueban el año académico, y aún repitiendo tienen dificultades y siguen llevando cursos desaprobados, como también los hay aquellos que mantienen notas desaprobatorias en determinados cursos y finalizan el año académico con notas desaprobatorias. La Tiesa dimensiona el término rendimiento académico en dos aspectos: a) rendimiento académico en sentido amplio, que comprende los promovidos invictos y los no promovidos 0 no invictos y rendimiento académico en sentido estricto que se corresponde con los promedios finales.

En nuestro sistema educativo universitario, el rendimiento académico se mide generalmente en base a los promedios finales obtenidos en los cursos que componen un plan de estudios, el sistema de calificación peruano es vigesimal ( 0 a 20 ), la nota aprobatoria es de 11 a 20 y la desaprobatoria oscila de 0 a 10 .El término reprobación alude al alumno que no obtiene una calificación de aprobado en una asignatura o en un examen. Calificación de suspenso.

La repitencia se entiende como la acción de cursar reiterativamente una actividad docente, sea por mal rendimiento del estudiante o por causas ajenas al ámbito académico .Como lo indica la experiencia, es particularmente difícil disponer de datos completos y confiables que permitan establecer indicadores de amplio espectro sobre la repitencia cuando hay curriculo flexible. De ahí que comúnmente se acepte el atraso escolar como un indicador proxy de la repitencia. En la Facultad de Obstetricia la nota requerida para aprobar una asignatura es de 11 puntos. Asimismo, el término deserción, en el ámbito educativo, se interpreta como el hecho de que habiéndose matriculado no inicia estudios en ningún curso o que habiendo iniciado los estudios los abandona

\section{MATERIAL YMÉTODOS}

El estudio se realizó durante el año académico 2006 en la Facultad de Obstetricia. La población objeto de estudio fueron 235 y 241 expedientes académicos de los estudiantes matriculados en el año 2004 y 2005, respectivamente.

\section{El procedimiento de recolección consistió en}

Recopilar la información desde una fuente secundaria, ya que se solicitaron los registros de notas y documentos curriculares a la Oficina de Registro Académico de la FAOB. 
El trabajo de campo se centró en recoger los datos de las siguientes variables:

- Alumnos matriculados por año académico.

- Características generales : edad y sexo.

- Promedio de notas por alumno matriculado.

- Asignaturas reprobadas por alumno.

- Índice de promovidos Invictos.

- Repitentes.

- Indice de reservas de matricula

- Indice de deserción.

\section{RESULTADOS, ANÁLISIS Y DISCUSIÓN}

Gráfico $N^{\circ}$ 01: Facultad de Obstetricia de la UNJBG de Tacna. Estudiantes según el Promedio Anual obtenido en el Año Académico 2004

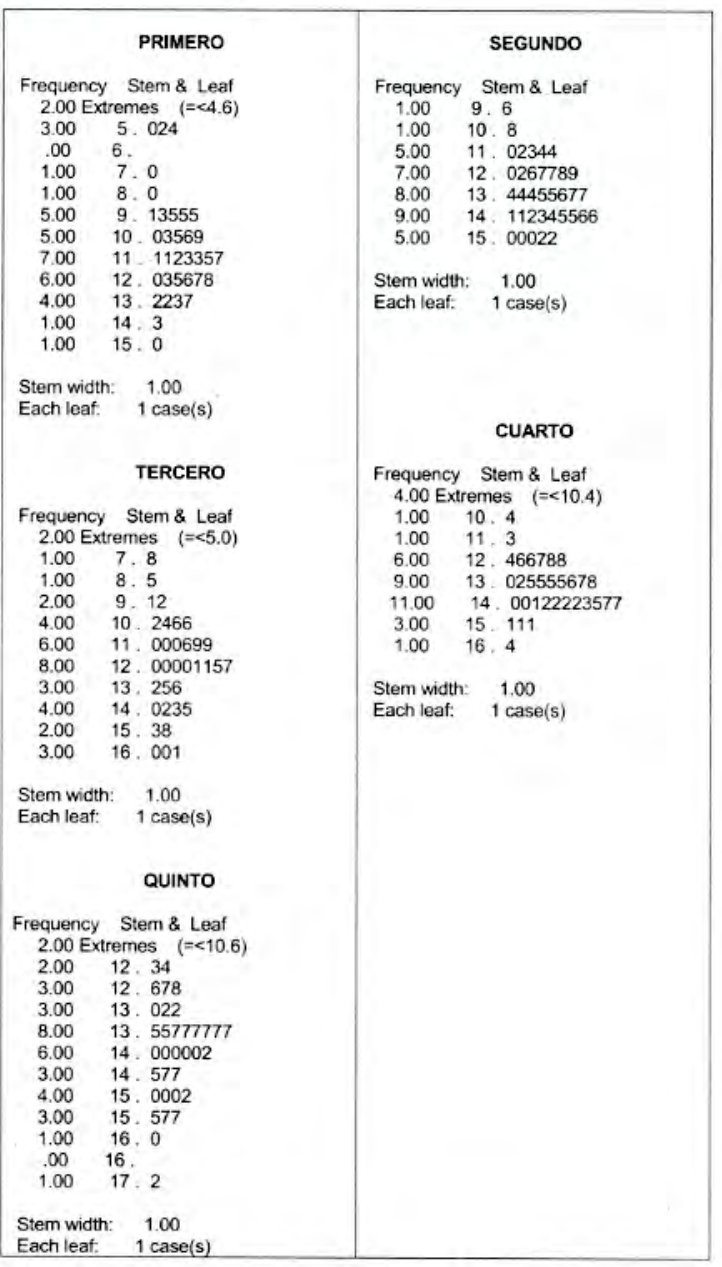

El Gráfico Nº1 nos permite apreciar que el menor promedio académico anual obtenido en el Año Académico 2004 es de 5,0 y corresponde al primer año de estudios, mientras que el promedio más alto es de 17,2 y se ubica en el quinto año de estudios; sin embargo, se aprecian dos valores extremos en el caso de primer año con promedios menores de 4,6. También se evidencia que la distribución de los datos forman una curva normal en el caso de primero. segundo, tercero y quinto año de estudios, a diferencia de cuarto año en que los datos forman una curva leptokúrtica. En el caso de segundo año, la curva presenta ligeramente un sesgo negativo, lo que denota que los promedios tienden a ser menores.
Tabla N 01: Facultad de Obstetricia de la UNJBG de Tacna. Estudiantes según Medidas Descriptivas de los Promedios Anuales porAño de Estudios, Año Académico 2004.

\begin{tabular}{|c|c|c|c|c|c|c|}
\hline & & \multicolumn{5}{|c|}{ ANOO DE ESTUDIOS } \\
\hline & & PRIMERO & SEGUNDO & TERCERO & CUARTO & QUINTO \\
\hline \multirow[t]{2}{*}{$\bar{N}$} & Valios & 37 & 39 & 40 & 39 & 36 \\
\hline & Perdidos & 3 & 1 & 0 & 1 & 4 \\
\hline Media & & 10.3797 & 13.1703 & 12.0177 & 132733 & 13.8328 \\
\hline Mediana & & 11.1500 & 13,4600 & 12.0000 & 13.6700 & 13.8750 \\
\hline Moda & & 9.55 & $14.17^{\mathrm{a}}$ & 12.00 & 1422 & 13.75 \\
\hline Desv. tip. & & 29897 & 1.4614 & 260500 & 1.7058 & 1.737 \\
\hline Asimetria & & -964 & .438 & .855 & -1.116 & -1.888 \\
\hline Minimo & & 236 & 967 & 4.45 & 820 & 671 \\
\hline Máximo & & 1509 & 1525 & 16.13 & 16.44 & 17.25 \\
\hline \multirow[t]{3}{*}{ Percentiles } & 25 & 92800 & 11.8600 & 10.725 & 12.6700 & 13.2125 \\
\hline & 50 & 11.1500 & 13.4600 & 12.0000 & 13.6700 & 13.8750 \\
\hline & 75 & 12.6100 & 14,4200 & 13.9085 & 14.3300 & 14.9375 \\
\hline
\end{tabular}

Fuente: Registro Académico de la FAOB

Gráfico No 02: Facultad de Obstetricia de la UNJBG de Tacna Estudiantes según el promedio anual obtenido en el AñoAcadémico 2004.

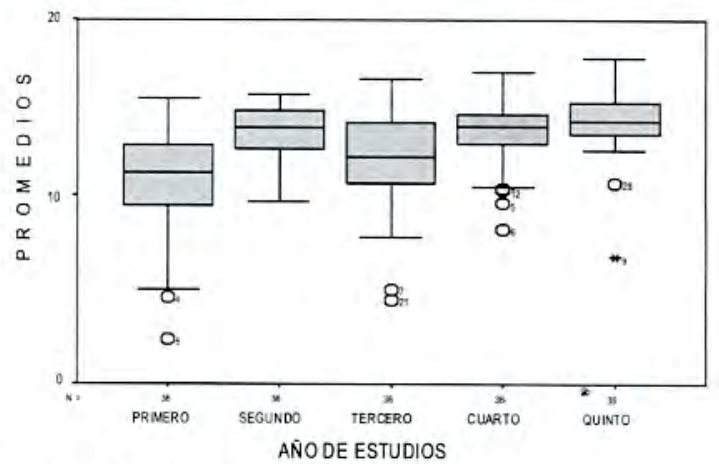

Fuente: Registro Académico de la FAOB

En la Tabla 1 y en el Gráfico 2 se visualiza que el promedio de quinto año en el año 2004 es el más alto, con un valor de 13,83 puntos , seguido de cuarto año , que presenta un promedio de 13,27 , similar valor presenta segundo año con un promedio de 13,17 , a diferencia de tercero que presenta un promedio de 12,01 y primer año que muestra el promedio más bajo con 11 puntos, valorados en la escala vigesimal de 0 a 20 puntos. La longitud de las cajas nos permite apreciar que los valores se encuentran más dispersos en las aulas.de primero y tercer año de estudios, y más concentrados en las aulas de quinto, segundo y cuarto, siendo este último año el que presenta menor dispersión.

Asimismo, se consideraron cuatro categorias para evaluar el rendimiento académico: Muy bueno: 17,00 -20,00, Bueno:14,00 - 16,99, Regular : 11,00 - 13,99,Deficiente $00,00-10,99$ puntos, respectivamente.

Los promedios por año de estudios se ubican en la categoría de bueno para el aula de quinto año, y regular para las restantes. Sin embargo, si se hace una valoración global respecto al promedio del año académico 2004 (12,53), éste se ubica en el rango de regular. Si se compara estos resultados con los obtenidos por Alvarez y Cols. al evaluar el rendimiento global de las promociones correspondientes a los años académicos 1998 (12) , 1999 (12,28), $2000(12,82)$, $2001(13,03), 2002(13,07)$ y 2003 (12,95) apreciamos que en todos los casos se ubican en la categoría de regular, la que no resulta satisfactorio académicamente de ningún modo. 


\section{CONCLUSIONES}

1. El nivel de rendimiento académico de la Facultad de Obstetricia en el año académico 2004, valorado según el promedio global por año, fue de 12,53 puntos, valor que se ubica en la categoria de regular( 11,00 - 13,99 puntos).

2. Las asignaturas más reprobadas por los alumnos/as de la FAOB en el Año Académico 2004 corresponden a los cursos de formación general, que se ubican en el primer año de estudios, y en las asignaturas de Matemática Aplicada , Histologia, Biología Celular y Molecular, Química Orgánica y Bioquímica ( $80 \%$ ), en segundo año los cursos de Psicología Evolutiva y Salud Mental asi como Microbiología y Parasitologia representan el $36,11 \%$ del total de cursos reprobados. En el tercer año, el $75 \%$ de los cursos reprobados corresponden a las materias de Farmacología General y Obstétrica, Propedéutica Obstétrica y Neonatología y Pediatría; asimismo, en cuarto y quinto año las materias más reprobadas corresponden a los cursos de Psicoprofilaxis Obstétrica y Seminario de Tesis con un 41,67 $\%$ y $83,33 \%$ respectivamente.

3. El índice puntual de deserción estudiantil tiene una frecuencia de 06 estudiantes en el Año Académico 2004.

4. El indice de estudiantes matriculados ( primero a quinto año de estudios) en la FAOB en el Año Académico 2004 fue de 202 estudiantes.

\section{BIBLIOGRAFÍA}

Álvarez Becerra, Rina; Acurio Caceres, Gladis; Villegas De Olazabal, Oscar; Nivel de Rendimiento Académico y Deserción Estudiantil en la Facultad de Obstetricia 19982003. Tacna, Perú, 2005.

Gonzales Fiegehen, Luis Eduardo. Repitencia y Deserción Universitaria en América Latina. . Informe sobre la Educación Superior en América Latina y el Caribe 20002005.IESALC.UNESCO.2006

La Tiesa , Margarita, La Deserción Universitaria, Edit. Universidad de Granada, España, 1997.

López Maquera, Miriam; Mattos Peña, Mario, Rendimiento Académico de la Facultad de Obstetricia. 19811997. UNJBG.

Salcedo V., Alberto. Métodos Estadisticos. Edit. CONCYTEC.Lima-Perú, 1990.

Alvarez Esteban, Ramón [en línea] www.unileon.es/temario.php?cod $=0507004$ [Consulta: 25.03.07]

Gómez C ,I. Análisis del grado de reprobación de la carrera de Ingenieria Comercial de la Universidad Austral de Chile [en Línea]www.ucsm.edu.pe/rabarcaf/vonue.html. [Consulta: 15.01.06].

\section{ANEXOS}

Cuadro $N^{\circ}$ 01: FACULTAD DE OBSTETRICIA DE LA UNJBG DE TACNA Estudiantes por año de estudios y según resultados del Año Académico 2004

\begin{tabular}{|c|c|c|c|c|c|c|c|c|c|c|}
\hline \multirow{2}{*}{\multicolumn{2}{|c|}{$\begin{array}{l}\text { AÑOS DE } \\
\text { ESTUDIOS }\end{array}$}} & \multicolumn{8}{|c|}{ HISTORIA ESTADISTICA DEL AÑO ACADEMICO 2004} & \multirow[b]{2}{*}{ Total } \\
\hline & & \multirow{2}{*}{$\begin{array}{r}\text { Invicta } \\
11\end{array}$} & \multirow{2}{*}{\begin{tabular}{|c|}
$\begin{array}{c}\text { Promovida } \\
\text { con } 1 \text { curso }\end{array}$ \\
6 \\
\end{tabular}} & \multirow{2}{*}{\begin{tabular}{|c|}
$\begin{array}{c}\text { Promovida } \\
\text { con } 2 \text { cursos }\end{array}$ \\
8 \\
\end{tabular}} & \multirow{3}{*}{\begin{tabular}{|c|}
$\begin{array}{c}\text { Promovida } \\
\text { con } 3 \text { cursos }\end{array}$ \\
1 \\
$2,4 \%$ \\
\end{tabular}} & \multirow{3}{*}{$\begin{array}{r}\text { Repitente } \\
11 \\
26,8 \%\end{array}$} & \multirow[t]{3}{*}{ Desertores } & \multirow[t]{3}{*}{$\begin{array}{c}\text { Traslado } \\
\text { interno } \\
\text { excepcional }\end{array}$} & \multirow{3}{*}{\begin{tabular}{|r|}
$\begin{array}{c}\text { Reserva de } \\
\text { matricula }\end{array}$ \\
4 \\
$9,8 \%$
\end{tabular}} & \\
\hline Primero & $\mathrm{N}^{0}$ & & & & & & & & & 41 \\
\hline & $\%$ & $26,8 \%$ & $14,6 \%$ & $19,5 \%$ & & & & & & $100,0 \%$ \\
\hline \multirow[t]{2}{*}{ Segundo } & $\mathrm{N}^{0}$ & 23 & 7 & 5 & 2 & 1 & & 1 & 2 & 41 \\
\hline & $\%$ & $56,1 \%$ & $17,1 \%$ & $12,2 \%$ & $4,9 \%$ & $2,4 \%$ & & $2,4 \%$ & $4,9 \%$ & $100,0 \%$ \\
\hline \multirow[t]{2}{*}{ Tercero } & $\mathrm{N}^{\circ}$ & 27 & 4 & 3 & 1 & 4 & & 1 & & 40 \\
\hline & $\%$ & $67,5 \%$ & $10,0 \%$ & $7,5 \%$ & $2,5 \%$ & $10,0 \%$ & & $2,5 \%$ & & $100,0 \%$ \\
\hline \multirow[t]{2}{*}{ Cuarto } & $\mathrm{N}^{0}$ & 32 & 5 & 1 & & 1 & & & 2 & 41 \\
\hline & $\%$ & $78,0 \%$ & $12,2 \%$ & $2,4 \%$ & & $2,4 \%$ & & & $4,9 \%$ & $100,0 \%$ \\
\hline \multirow[t]{2}{*}{ Quinto } & $\mathrm{N}^{0}$ & 34 & 1 & 2 & & & 1 & & 1 & 39 \\
\hline & $\%$ & $87,2 \%$ & $2,6 \%$ & $5,1 \%$ & & & $2,6 \%$ & & $2,6 \%$ & $100,0 \%$ \\
\hline \multirow[t]{2}{*}{ Total } & $\mathrm{N}^{0}$ & 127 & 23 & 19 & 4 & 17 & 1 & 2 & 9 & 202 \\
\hline & $\%$ & $62,9 \%$ & $11,4 \%$ & $9,4 \%$ & $2,0 \%$ & $8,4 \%$ & $0,5 \%$ & $1,0 \%$ & $4,5 \%$ & $100,0 \%$ \\
\hline
\end{tabular}

Fuente: Oficina de Registro Académico de la FAOB 
Cuadro N 02: FACULTAD DE OBSTETRICIA DE LA UNJBG DE TACNA Estudiantes por año de estudios y según resultados del Año Académico 2005

\begin{tabular}{|c|c|c|c|c|c|c|c|c|c|c|}
\hline \multirow{2}{*}{\multicolumn{2}{|c|}{$\begin{array}{l}\text { AÑOS DE } \\
\text { ESTUDIOS }\end{array}$}} & \multicolumn{8}{|c|}{ HISTORIA ESTADISTICA DEL AÑO ACADEMICO 2005} & \multirow{4}{*}{\begin{tabular}{|r|} 
Total \\
64 \\
$100,0 \%$
\end{tabular}} \\
\hline & & \multirow{2}{*}{$\begin{array}{r}\text { Invicta } \\
22\end{array}$} & \multirow{2}{*}{\begin{tabular}{|c|}
$\begin{array}{c}\text { Promovido } \\
\text { con } 1 \text { curso }\end{array}$ \\
11 \\
\end{tabular}} & \multirow{2}{*}{\begin{tabular}{|c|}
$\begin{array}{c}\text { Promovido } \\
\text { con } 2 \text { cursos }\end{array}$ \\
9 \\
\end{tabular}} & \begin{tabular}{|l|} 
Promovido \\
con 3 cursos \\
\end{tabular} & \multirow{2}{*}{$\begin{array}{c}\text { Repitente } \\
11\end{array}$} & \multirow{2}{*}{$\begin{array}{c}\text { Desertores } \\
5\end{array}$} & \multirow{3}{*}{\begin{tabular}{|c|}
$\begin{array}{c}\text { Traslado } \\
\text { interno } \\
\text { excepcional }\end{array}$ \\
2 \\
$3,1 \%$ \\
\end{tabular}} & \begin{tabular}{|c|}
$\begin{array}{c}\text { Reserva de } \\
\text { matricula }\end{array}$ \\
\end{tabular} & \\
\hline Primero & $\mathrm{N}^{\circ}$ & & & & 4 & & & & & \\
\hline & $\%$ & $34,4 \%$ & $17,2 \%$ & $14,1 \%$ & $6,3 \%$ & $17,2 \%$ & $7,8 \%$ & & & \\
\hline Segundo & $\mathrm{N}^{0}$ & 9 & 10 & 4 & & 1 & & & & 24 \\
\hline & $\%$ & $37,5 \%$ & $41,7 \%$ & $16,7 \%$ & & $4,2 \%$ & & & & $100,0 \%$ \\
\hline Tercero & $\mathrm{N}^{0}$ & 24 & 9 & 4 & & 1 & & 1 & 2 & 41 \\
\hline & $\%$ & $58,5 \%$ & $22,0 \%$ & $9,8 \%$ & & $2,4 \%$ & & $2,4 \%$ & $4,9 \%$ & $100,0 \%$ \\
\hline Cuarto & $\mathrm{N}^{0}$ & 30 & 2 & 1 & & 1 & & 1 & 1 & 36 \\
\hline & $\%$ & $83,3 \%$ & $5,6 \%$ & $2,8 \%$ & & $2,8 \%$ & & $2,8 \%$ & $2,8 \%$ & $100,0 \%$ \\
\hline Quinto & $\mathrm{N}^{0}$ & 37 & 1 & 2 & & 1 & & & & 41 \\
\hline & $\%$ & $90,2 \%$ & $2,4 \%$ & $4,9 \%$ & & $2,4 \%$ & & & & $100,0 \%$ \\
\hline Total & $\mathrm{N}^{0}$ & 122 & 33 & 20 & 4 & 15 & 5 & 4 & 3 & 206 \\
\hline & $\%$ & $59,2 \%$ & $16,0 \%$ & $9,7 \%$ & $1,9 \%$ & $7,3 \%$ & $2,4 \%$ & $1,9 \%$ & $1,5 \%$ & $100,0 \%$ \\
\hline
\end{tabular}

Fuente: Oficina de Registro Académico de la FAOB 